- Revista de Iniciação à Docência, v. 5, n. 3, 2020 -

Publicação: março, 2021 - ISSN 2525-4332

\title{
MONITORIA ACADÊMICA E O AUXÍLIO AO ENSINO-APRENDIZAGEM EM UM CURSO DE CIÊNCIAS BIOLÓGICAS
}

Renata Fernandes de Matos ${ }^{1}$

\begin{abstract}
RESUMO: O estudo analisa as contribuições das atividades de monitoria acadêmica para o processo de ensino-aprendizagem em um curso de Ciências Biológicas. Seu desenvolvimento se deu com base em uma pesquisa do tipo qualitativa por meio do método descritivo. O público-alvo foram os alunos de um curso de Licenciatura em Ciências Biológicas de um campus interiorano vinculado à Universidade Estadual do Ceará. A coleta de dados se deu por meio da aplicação de questionários, tomando-se como grupo pesquisado 90 alunos distribuídos em todos os semestres do curso. Pelos resultados obtidos na análise destaca-se que a monitoria proporciona valiosas contribuições para o ensino-aprendizagem, contando os alunos com um auxílio recorrente, sobretudo, para questionamentos de maiores proporções. Os alunos têm avaliado positivamente o trabalho desenvolvido pelos monitores, destacando que se sentem confortáveis em serem acompanhados por eles, o que tem como resultado a demanda da monitoria para uma série de disciplinas ao longo do curso. Conclui-se assim que a monitoria é uma atividade viável para o desenvolvimento do ensino-aprendizagem no âmbito das Ciências Biológicas.
\end{abstract}

Palavras-Chave: Estratégias de Ensino; Ensino de Ciências; Formação Docente; Licenciatura.

ABSTRACT: The study analyzes the contributions of academic monitoring activity to the teachinglearning process in a Biological Sciences course. Its development was based on qualitative research using the descriptive method. The target audience was students of a Biological Sciences Degree course from an inland campus linked to the State University of Ceará. Data collection took place through the application of questionnaires, taking as a researched group 90 students distributed in all semesters of the course. Based on the results obtained in the analysis, it is highlighted that monitoring provides valuable contributions to teaching and learning, with students receiving recurrent assistance, above all, for questions of greater proportions. Students have positively evaluated the work developed by the monitors, highlighting that they feel comfortable being accompanied by them, which results in the demand for monitoring for a series of disciplines throughout the course. It is concluded that monitoring is a viable activity for the development of teaching learning in the scope of Biological Sciences.

Key words: Teaching Strategies; Science Teaching; Teacher Education; Graduation.

\section{Introdução}

A monitoria acadêmica é uma atividade que vem sendo praticada desde a antiguidade, onde as escolas medievais do século XII e XIII contavam com os chamados "repetidores" que desempenhavam funções semelhantes ao que hoje é praticado pelos monitores (MENZEL et al., 2015). Esta atividade pode ser definida como uma ferramenta de auxílio ao processo de ensino-aprendizagem, contribuindo com o trabalho dos docentes e facilitando a aquisição de conhecimentos pelos discentes (NATÁRIO; SANTOS, 2010).

A monitoria é uma estratégia de melhoria do ensino ofertada pelas instituições de nível superior, tendo como foco os cursos de graduação. Seu objetivo é incentivar os

\footnotetext{
${ }^{1}$ Professora da Faculdade de Educação, Ciências e Letras de Iguatu/CE. Universidade Estadual do Ceará. Doutora em Agronomia/Fitotecnia. E-mail: <renatafmatos@hotmail.com>.
} 
monitores a auxiliar os professores no que diz respeito a ampliação da acessibilidade dos conteúdos aos alunos monitorados, assim como a ampliação do campo de visão desses sobre a disciplina (FARIAS, 2013). Enquanto atividade acadêmica, deve ocorrer em consonância com o projeto político-pedagógico adotado pelo curso ao qual está vinculado (FERNANDES et al., 2015).

Com o desenvolvimento dos programas de monitoria uma significativa melhoria do processo de ensino-aprendizagem tem sido observada, possibilitando um melhor entendimento dos conteúdos e resultando em contribuições diferenciadas para o rendimento dos alunos nas disciplinas (NETO; PARENTE; FRAGA, 2019). Dessa forma, a monitoria representa uma perspectiva concreta para o fortalecimento do conhecimento no nível superior, o que está associado à qualificação técnico-científica apresentada pelos seus condutores (FERNANDES et al., 2015).

As atividades da monitoria devem ser desenvolvidas mediante o estabelecimento de novas práticas e experiências pedagógicas que visem fortalecer a articulação entre teoria e prática e a integração curricular em seus diferentes aspectos (UNIDERP, 2019). Sua execução desperta nos alunos o interesse pela docência mediante o desempenho de atividades ligadas ao ensino, possibilitando a participação na organização e na condução das disciplinas, o que terá por resultado a melhoria no desenvolvimento de habilidades didáticas (LINS, 2007).

O desenvolvimento de um programa de monitoria promove uma espécie de cooperação mútua entre discentes e docentes, possibilitando ao aluno-monitor a vivência com o professor e como as atividades técnico-didáticas por ele executadas (UNIDERP, 2019). Dessa forma, a monitoria passa a ser considerada um instrumento capaz de facilitar o trabalho realizado pelos professores, contribuindo com o ensino na medida em que os monitores proporcionam aos alunos monitorados uma maior experiência com 0 aprendizado (SILVA; BELO, 2012).

É necessário, entretanto, que para o bom desenvolvimento de suas competências, os monitores recebam orientações adequadas quanto ao trabalho que devem executar, o que terá como reflexo a promoção de um ambiente de aprendizagem construtivo e gratificante (NATARIO, 2001). Com isto, a monitoria promove o desenvolvimento de diversas aptidões nos alunos-monitores, contribuindo para torná-los profissionais preparado para os desafios da profissão frente às exigências do mercado (VICENZI, 2016).

Nessa perspectiva, os alunos-monitores atuam como mediadores da aprendizagem, transferindo conhecimentos complementares e atuando como um suporte para as demandas acadêmicas (FIGUEIREDO; FILIPPIN; VENDRUSCULO, 2016). Por esta realidade, torna-se possível promover uma aproximação dos monitores com um ensino precoce, resultando em sua preparação para a superação de dificuldades no campo do ensino (ABREU, 2014). 
Para que as atividades de monitoria sejam então eficientes, torna-se necessário uma maior valorização dos trabalhos que vem sendo desenvolvidos pelos monitores, o que deve ser sustentado por sua relação com os professores, tendo como resultado o desenvolvimento satisfatório dos graduandos (BENIGNO et al., 2012). Dessa forma, é possível observar um fortalecimento da interação aluno-monitor-professor, proporcionando a todos trocas mais intensas de conhecimentos e experiências (BOTELHO et al., 2019).

Neste sentido, o presente artigo tem por objetivo analisar as contribuições que a monitoria acadêmica proporciona ao processo de ensino-aprendizagem em um curso de Ciências Biológicas.

\section{Metodologia}

A pesquisa foi desenvolvida com base nos métodos qualitativos, o qual possibilitam a obtenção de conclusões amplas e possibilitam uma descrição detalhada do objeto de estudo (SCHNEIDER; FUJII; CORAZZA, 2017). Adotou-se também o caráter descritivo para o estudo, buscando demonstrar as características de uma população ou de um grupo de indivíduos, sendo, para isto, necessário o levantamento das opiniões dos envolvidos nos processos de monitoria (GIL, 2010).

O desenvolvimento da pesquisa se deu no ano de 2019, sendo investigado um curso de Licenciatura em Ciências Biológicas pertencente a Universidade Estadual do Ceará, estando o mesmo vinculado a uma das unidades do interior da presente instituição. Ao todo foram entrevistados 90 alunos, tomando-se uma amostra de 10 alunos de cada semestre, o que possibilitou obter informações sobre a monitoria em todo o curso.

A coleta dos dados se deu por meio da aplicação de questionários, podendo estes ser definidos como uma técnica de investigação composta por um conjunto de questões que são submetidas aos indivíduos com o propósito de obter informações (GIL, 2010). A análise dos resultados ocorreu de acordo com as premissas da estatística descritiva, bem como mediante a categorização de informações segundo metodologia proposta por Bardin (2011).

Toda a pesquisa foi desenvolvida de acordo com os aspectos éticos e legais que o Conselho Nacional de Saúde (CNS) propõe, seguindo para isto as pressuposições da lei 510/2016 (BRASIL, 2016). Em vias de legalizar a coleta dos dados, os alunos maiores de idade assinaram um Termo de Consentimento Livre e Esclarecido, e os alunos menores de idade requisitaram aos seus pais ou responsáveis que assinassem um Termo de Assentimento Livre e Esclarecido, permitindo a utilização das informações coletadas.

\section{Resultados e discussão}

O trabalho desenvolvido pelos monitores nos diversos cursos de graduação tem contribuído para a melhoria dos processos de ensino-aprendizagem em diversas áreas do conhecimento. Para os cursos de Ciências Biológicas, uma atenção especial deve ser dada 
à prática da atividade, pois, diante das diferentes disciplinas ofertadas, dificuldades podem comprometer o aprendizado dos alunos, requerendo apoio mais sistemático no que diz respeito ao fortalecimento do ensino.

Para que o aprendizado nos cursos de nível superior seja de fato efetivo é necessário que os programas de monitoria forneçam um suporte adequado para que os alunos solidifiquem os conteúdos estudados em sala de aula. Nesse contexto, o planejamento das atividades a serem executadas durante a monitoria deve ser realizado pelos monitores em conjunto com os professores, o que se configura como um espaço para a formação e crescimento de ambos (NUNES, 2007).

Os professores atuam como os primeiros transmissores do conhecimento, estando em contato com os alunos em sala de aula e, dependendo da disciplina, em aulas de laboratório e campo. Já os monitores, em concordância com o que está sendo estudado em sala de aula, promovem atividades extras que visam complementar o conhecimento inicial adquirido, retirando dúvidas dos alunos e dando suporte para as suas diversas necessidades.

Contudo, diante do surgimento de dúvidas, é comum os alunos buscarem meios para solucioná-las antes de recorrerem aos serviços dos monitores ou professores. Nessa perspectiva, buscou-se investigar a quem os alunos recorrem quando apresentam alguma dúvida, tanto no que diz respeito a uma demanda inicial, muitas vezes relacionada a questões simples, quanto a uma demanda posterior, onde a dúvida perdura por não ter sido efetivamente esclarecida.

Conforme pode ser observado na Tabela 1, a maioria dos alunos afirmou recorrer inicialmente aos próprios colegas quando apresentam alguma dúvida, o que foi indicado por $80 \%$ dos entrevistados. Esse resultado mostra que os alunos veem nos colegas uma oportunidade de auxílio ao aprendizado, o que é justificado por estarem cursando a mesma disciplina e terem acesso aos mesmos conteúdos, podendo tê-los entendido completamente e estarem aptos a esclarecer possíveis dúvidas.

Tabela 1 - A quem os alunos recorrem quando apresentam dúvidas nas disciplinas.

\begin{tabular}{cccc}
\hline Inicialmente & Indicação & Posteriormente & Indicação \\
\hline Colegas & $80 \%$ & Colegas & $0 \%$ \\
Monitores & $20 \%$ & Monitores & $70 \%$ \\
Professores & $0 \%$ & Professores & $30 \%$ \\
\hline
\end{tabular}

Fonte: Autora

Essa busca pelos colegas pode também ser justificada pelo fato dos alunos se sentirem confortáveis em expressar suas dúvidas para indivíduos que apresentam um nível de instrução igual ao seu, superando os bloqueios que podem existir na busca pelo auxílio de pessoas com maior grau de instrução. Somado a isto, muitas vezes essas dúvidas se referem a questões simples, as quais podem ser esclarecidas pelos colegas ou mesmo por uma revisão mais atenciosa do conteúdo visto em sala de aula. 
Os 20\% restante dos alunos afirmou que quando apresentam alguma dúvida já recorrem aos monitores para obter seu esclarecimento. Mesmo sendo considerada uma porcentagem baixa de estudantes participantes da pesquisa, fica visível o quão importante é o trabalho realizado pelos monitores no curso em questão, atuando no suporte direto às demandas dos alunos e contribuindo para a superação das dificuldades no ensino (ABREU, 2014).

Para o esclarecimento inicial das dúvidas, constatou-se que nenhum aluno faz a busca pelo professor, o que mostra que, com exceção dos colegas, os monitores são os únicos agentes de auxílio ao aprendizado nessa etapa. A ajuda dos monitores ressalta-se por estes serem alunos que estão em processo de formação, indicando que o alunomonitor primeiro aprende para depois ensinar, e novamente aprende ensinando, o que é um dos objetivos da monitoria (JESUS, et al., 2012).

Apesar da baixa porcentagem de procura dos alunos pelos monitores, quando se trata do esclarecimento inicial das dúvidas, ao se considerar a busca por um esclarecimento posterior, mais aprofundado, o cenário observado passa a ser bem diferente. Para esta situação, os colegas já não são mais consultados como anteriormente, porém, a busca pelos monitores assume uma proporção na ordem de $70 \%$, indicando o quão relevante passa a ser o trabalho executado por estes.

Isto se dá porque os alunos reconhecem que, mesmo contando com a ajuda dos colegas, é necessário fazer a busca por um conhecimento mais preciso, necessitando das instruções de indivíduos preparados para isto. Os monitores surgem assim como peças estratégicas para avanços no processo de aprendizado e formação, sem os quais um possível déficit no rendimento dos alunos seria uma realidade em diversas instituições de ensino.

Para a situação em questão, a busca pelos professores para o esclarecimento de dúvidas passou a ser indicada por apenas 30\% dos alunos entrevistados, o que mostra que mesmo contando com a ajuda dos monitores, alguns alunos ainda se sentem mais seguros recorrendo aos professores. Contudo, apesar do viés favorável em se contar com o auxílio extraclasse dos professores, a busca pelos monitores instiga desafios e cria oportunidades para o crescimento destes, o que refletirá em benefícios para a própria disciplina.

Com isto, os programas de monitoria não estão voltados apenas para a melhoria do desempenho dos discentes no âmbito acadêmico, mas também para a criação de oportunidades para a atuação de alunos-monitores no campo da docência, estreitando os vínculos destes com a universidade (JESUS et al., 2012). Esta implicação é ainda mais importante quando tratamos dos cursos de licenciatura, pois, os alunos conseguem adquirir uma maior experiência devido a autonomia que adquirem na condução da monitoria.

A atuação dos monitores é assim vista como um ponto chave para a mudança na realidade do aprendizado em muitas instituições de ensino. Dependendo da disciplina em 
que os alunos estão matriculados, conteúdos mais difíceis de serem assimilados podem ser observados, o que se soma ao fato de alguns alunos terem maiores dificuldades na compreensão dos assuntos, conferindo importantes desafios a serem encarados pelos monitores.

Porém, independentemente do nível de dificuldade encontrado pelos monitores, um bom trabalho deve ser realizado por eles, de forma a ofertar aos alunos monitorados a melhor qualidade de acompanhamento possível. Nesse contexto, buscou-se saber dos alunos entrevistados quais notas eram atribuídas ao trabalho que desenvolvido pelos monitores dentro do curso de Ciências Biológicas, podendo os resultados ser observados no Gráfico 1.

Gráfico 1 - Nota que os alunos atribuem às atividades realizadas pelos monitores.

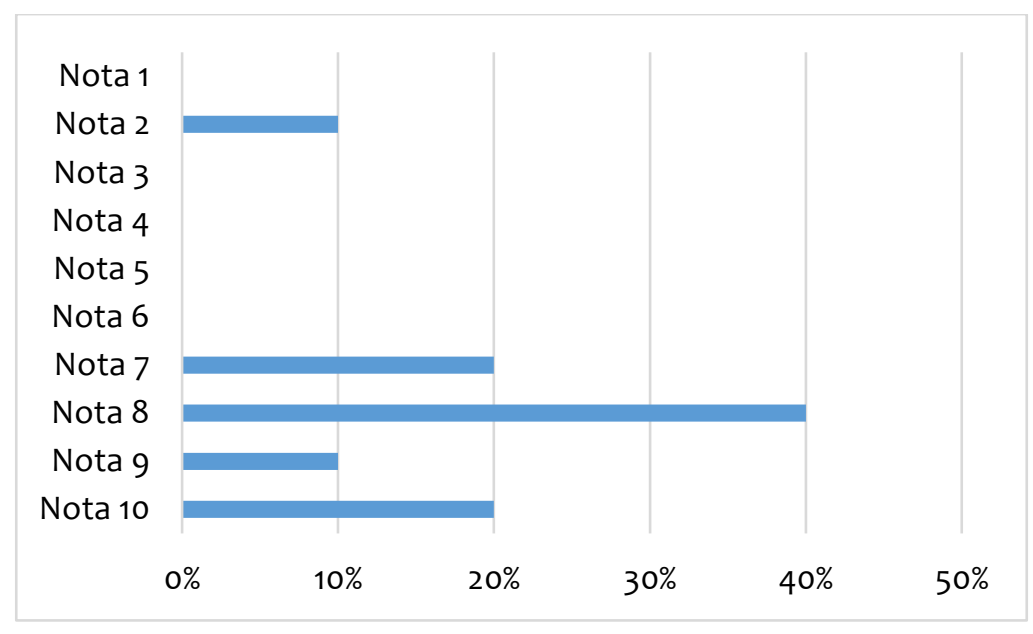

Fonte: Autora

É possível perceber que a maioria dos entrevistados (90\%) indicaram nota igual ou acima de sete para os monitores, o que mostra que o trabalho realizado por estes foi bem avaliado pelos seus principais beneficiados. Isto é confirmado pelo fato da nota 10 ter sido atribuída por $20 \%$ do público-alvo desta pesquisa, mostrando que para estes estudantes os trabalhos de monitoria estão bem próximos do que poderia ser considerado perfeito. Entretanto, é notável que 10\% dos entrevistados apontaram nota dois nesta avaliação, o que deve ser observado considerando sua grande discrepância em relação a todas as outras notas indicadas pelo restante dos participantes. Os alunos que fizeram tal indicação provavelmente tiveram experiências com a monitoria em uma mesma disciplina, onde, dependendo da condução que foi dada pelo monitor, os trabalhos podem não ter sido realizados adequadamente ou os alunos podem não ter atribuído a devida importância às atividades desenvolvidas.

A falta de interesse de alguns alunos pode ser um fator comprometedor para a boa execução de um programa de monitoria acadêmica. Matoso (2014), ao conduzir pesquisas sobre a monitoria acadêmica, concluiu que a principal dificuldade enfrentada é a falta de interesse dos discentes monitorados. Por vezes, eles não percebem a monitoria como uma 
forma de auxílio ao aprendizado. Alunos nesta situação não dão importância a atividade e, por consequência, não avaliam bem sua execução.

Faz-se necessário então uma investigação sobre as experiências que os alunos têm vivenciado com as atividades executadas pela monitoria. Nesse contexto, buscou-se obter dos alunos relatos sobre o auxílio que eles receberam dos monitores nas diferentes disciplinas do curso, destacando-se as falas a seguir:

$$
\begin{aligned}
& \text { "Me sinto mais à vontade com o monitor". } \\
& \text { "Com o auxílio do monitor consigo aprender mais". } \\
& \text { "O monitor oferece um ponto de vista diferente do professor". }
\end{aligned}
$$

“Os monitores, muitas vezes, apresentam uma linguagem mais acessível para o entendimento dos alunos".

“O monitor tem disponibilidade de tempo para acompanhar a turma em outros horários além da aula".

Pelos diferentes relatos apresentados, percebe-se a eficácia dos trabalhos de monitoria no que se refere ao incentivo e concretização da transmissão do conhecimento. Destaca-se para isto a grande proximidade existente entre monitores e alunos, o que culmina em uma integração pessoal e possibilita a melhoria do ensino-aprendizagem (SILVA; PAULINO; MACEDO, 2015).

Os alunos destacam que se sentem mais à vontade com os monitores, o que se dá por estes também serem alunos em processo de formação. Com isto, é possível vivenciar uma sensação de igualdade durante as aulas de monitoria, o que muitas vezes não é possível quando os educadores são os professores. Assim, confirma-se o que aponta Schmitt (2013), ao afirmar que a atuação dos monitores é útil para diminuir a ansiedade e o medo apresentado por muitos alunos.

Quando os alunos apontam que conseguem aprender de forma mais adequada os assuntos estudados com o auxílio dos monitores, fica evidente o potencial que a monitoria acadêmica oferece aos processos de aprendizagem. Esses ganhos de aprendizagem podem ser alcançados porque os monitores oferecerem aos alunos um ponto de vista diferente daquele apresentado pelos professores, além de trabalharem com uma linguagem mais acessível ao entendimento dos estudantes.

A melhoria no aprendizado é possível também devido a dedicação de tempo dos monitores, ressaltando aos alunos a importância do acompanhamento nos horários além das aulas. Assim, os alunos monitorados experimentam uma formação diferenciada e possibilidades mais amplas de conhecimentos (BOTELHO et al., 2019), além de absorverem melhor os conteúdos abordados (FURTADO; DUARTE; ALMEIDA, 2017).

As experiências positivas pelas quais os alunos passam quando são acompanhados por uma monitoria acadêmica possibilitam criar uma visão diferenciada sobre o ensino. Quando se obtém um bom rendimento em uma disciplina, é viável observar quais fatores contribuíram para o seu êxito, devendo estes ser replicados e adaptados às outras 
disciplinas, o que é amplamente viável quando se trata da monitoria acadêmica. Dessa forma, além possibilitar uma melhor formação, a monitoria desperta nos alunos interesse maior pela disciplina em curso (SOARES; SANTOS, 2008).

Com base na viabilidade e aplicabilidade da atividade de monitoria acadêmica, buscou-se saber dos alunos para quais disciplinas do curso de Ciências Biológicas estes demandavam sua execução, sendo possível obter as informações presentes no Quadro 1. As respostas apresentadas foram categorizadas de acordo com as áreas do conhecimento em que se enquadram no curso de Licenciatura em Ciências Biológicas.

Pelos resultados obtidos, observa-se que foram indicadas disciplinas de diversas áreas do conhecimento, mostrando que a monitoria é uma atividade amplamente demandada pelos alunos, devendo auxiliar os discentes durante o decorrer de todo o curso. Este comportamento deve ser visto como um suporte essencial para o complemento do aprendizado, elevando o desempenho observado em todas as disciplinas (FIGUEIREDO; FILIPPIN; VENDRUSCULO, 2016).

Quadro 1 - Disciplinas do curso de Ciências Biológicas que demandam monitoria.

\begin{tabular}{|c|c|}
\hline Área do conhecimento & Disciplinas indicadas \\
\hline Básico & Física \\
& Química Geral \\
Química Orgânica
\end{tabular}

Fonte: Autora

A demanda pela monitoria nas disciplinas do conhecimento básico, além de proporcionar base adequada para o seguimento do aprendizado nas disciplinas posteriores, ainda contribui para a superação de dificuldades oriundas do ensino médio. Isto é possível já que essas disciplinas, muitas vezes, contemplam assuntos que os alunos veem em momentos anteriores ao nível superior, os quais, quando não bem compreendidos, podem se tornar um fator comprometedor do aprendizado na graduação.

A busca pelo auxílio da monitoria na área pedagógica é algo que se ressalta nos cursos de licenciatura, tendo sido indicadas disciplinas de grande importância para o curso em questão. Contar com a ajuda dos monitores em disciplinas como Didática e Estágio Supervisionado é algo que pode resultar em uma maior segurança dos alunos quanto à 
prática da docência, o que muitas vezes causa um certo receio, mesmo estando os alunos nos cursos de licenciatura.

O mesmo pode ser inferido para as disciplinas da área molecular, as quais, por contarem com a interdisciplinaridade, muitas vezes, são vistas como de difícil compreensão. Dessa forma, adquirir uma base de conhecimentos adequada em uma disciplina, torna-se algo indispensável para o bom rendimento nas disciplinas posteriores, possibilitando um maior contato dos alunos com os conteúdos, além de proporcionar uma melhor assimilação dos conhecimentos que são exigidos durante as avaliações (SOUZA; GOMES, 2015).

Diferentes disciplinas que são específicas da Ciências Biológicas foram apontadas pelos alunos entrevistados. Entre as áreas demandadas, destaca-se a vegetal, animal e ambiental, demonstrando que a necessidade de auxílio ao aprendizado independe do conteúdo que é estudado, sendo a monitoria estratégica para auxiliar na compreensão de diversos conhecimento (SCHNEIDER, 2006).

Com isto, a melhoria do aprendizado nas diferentes áreas de um curso tem como resultado uma adequada formação acadêmica dos discentes, o que torna possível uma melhor preparação profissional, sobretudo, quando se trata de futuros professores. Dessa forma, a monitoria acadêmica proporciona benefícios a todos os seus envolvidos (NUNES, 2007), o que pode ser observado em curto e longo prazo.

\section{Considerações finais}

Por meio desse estudo infere-se que a monitoria acadêmica é atividade que tem contribuído diretamente para o fortalecimento do ensino-aprendizagem no âmbito das Ciências Biológicas. O suporte fornecido aos alunos proporciona maior segurança no que diz respeito ao preparo acadêmico, podendo os discentes recorrerem aos monitores sempre que apresentam dúvidas. Todo esse processo tem resultado em um melhor aprendizado e um adequado rendimento nas diversas disciplinas.

Com a avaliação dos trabalhos desenvolvidos pelos monitores é possível se ter um norte para as ações que estão em curso e para as que ainda serão desenvolvidas. Auxiliar alunos em processo de formação tem se configurado como uma oportunidade para a transformação do ensino no nível superior, o que tem por resultado o reconhecimento dos trabalhos executados, de tal sorte que os alunos valorizam o esforço, a dedicação e o empenho que vem sendo fornecido pelos monitores. Dessa forma, as experiências de sucesso vivenciadas com a monitoria acadêmica devem ser reproduzidas para outras disciplinas, como demandado pelos alunos na presente pesquisa. Assim, incentivar a aquisição de conhecimentos no meio acadêmico perpassa as atividades desenvolvidas em sala de aula, sendo necessário um suporte extraclasse continuado, para o qual a monitoria se encaixa e tem apresentado excelentes resultados. 


\section{Referências}

ABREU, T. O. A monitoria acadêmica na percepção dos graduandos de enfermagem. Revista de Enfermagem da UERJ, v. 22, n. 5, p. 507-512, 2014.

BARDIN, L. Análise de conteúdo. São Paulo: Edições 70. 2011. p. 229.

BENIGNO, A. P. A.; LIMA, A. C. S.; OLIVEIRA, E. C.; OLIVEIRA, P. C. C. A atividade de monitoria no ensino de Química: estudo de caso do IFAL Campus Murici. In: ENCONTRO NACIONAL DE ENSINO DE QUÍMICA; ENCONTRO DE EDUCAÇÃO QUÍMICA DA BAHIA, 16, 10, 2012. Anais... Salvador/BA: UFBA, 2012.

BOTELHO, L. V.; LOURENÇO, A. E. P.; LACERDA, M. G.; WOLLZ, L. E. B. Monitoria acadêmica e formação profissional em saúde: uma revisão integrativa. ABCS Health Sciences, v. 44, n. 1, 2019, p. 67-74.

BRASIL. Conselho Nacional de Saúde. Lei n 510 de 07 de abril de 2016. Brasília/DF, 07. dez. 2016.

FARIAS, J. P. A monitoria como prática colaborativa na Universidade. Dissertação de Mestrado. Programa de Pós-Graduação em Linguística Aplicada e Estudos da Linguagem. Pontifícia Universidade Católica de São Paulo. 2013.

FERNANDES, N. C.; CUNHA, R. R.; BRANDÃO, A. F.; CUNHA, L. L.; BARBOSA, P. D.; SILVA, C. O.; SILVA, M. S. A. Monitoria acadêmica e o cuidado da pessoa com Estomia: relato de experiência. Revista Mineira de Enfermagem, v. 19, n. 2, 2015, p. 238-241.

FIGUEIREDO, T. C.; FILIPPIN, N. T.; VENDRUSCULO, A. P. Percepção dos discentes de fisioterapia acerca da monitoria acadêmica. Fisioterapia Brasil, v. 17, n. 5, 2016, p. 450-456.

FURTADO, O. S.; DUARTE, A. F. V.; ALMEIDA, E. A.; OLIVEIRA, R. G. Contribuições da monitoria para desenvolvimento de habilidades e formação de competências pedagógicas. In: V Congresso Nacional de Educação, Recife/PE, 2017. Disponível em: <http://www.editorarealize.com.br/revistas/conedu/trabalhos/TRABALHO_EV117_MD1_SA16_ID5867_12092018173606.pdf>.

GIL, A. C. Como elaborar projetos de pesquisa. 5. ed. São Paulo/SP: Atlas, 2010.

JESUS, D. M. O.; MANCEBO, R. C.; PINTO, F. I. P.; BARROS, G. V. E. Programas de monitorias: um estudo de caso em uma IFES. Pensamento Contemporâneo em Administração, v. 6, n. 4. 2012, p. 61-86.

LINS, D. 2007. Ser Monitor. Faculdade Mauricio de Nassau.

Disponível em <http://mauriciodenassau.edu.br/artigo/exibir/cid>: Acesso em: 28 abr. 2020.

MATOSO, L. M. L. A importância da monitoria na formação acadêmica do monitor: um relato de experiência do monitor. Revista Científica da Escola da Saúde, v. 3, n. 2, 2014, p. 77-83.

MENZEL, T. C. Monitoria na área da zoologia de invertebrados: uma possibilidade de ensino e aprendizagem. Anais.... III CIECITEC, Santo Ângelo/RS, 2015, p. 10-12.

NATÁRIO, E. G.; SANTOS, A. A. A. Programa de monitores para o ensino superior. Estudo de Psicologia, v. 27, n. 3, 2010, p. 64-74.

NATÁRIO, E. G. Programa de Monitores para Atuação no Ensino Superior: Proposta de Intervenção. 142 f. Tese (Doutorado)- Faculdade de Educação, Universidade Estadual de Campinas, Campinas/SP, 2001. 
NETO, J. G. P.; PARENTE, N. N.; FRAGA, W. B. Uma análise das concepções discentes acerca da monitoria no curso de licenciatura em Física no IFCE. Revista Docência do Ensino Superior, v. 9, n. 1, 2019, p. 1-16.

NUNES, J. B. C. Monitoria acadêmica: espaço de formação. Natal/RN: EDUFRN, 2007, p. 45-58.

SCHNEIDER, M. S. P. S. Monitoria: instrumento para trabalhar com a diversidade de conhecimento em sala de aula. Revista Eletrônica Espaço Acadêmico, v. mensal, 2006, p. 65.

SCHNEIDER, E. M.; FUJII, R. A. X; CORAZZA, M. J. Pesquisas quali-quantitativas: contribuições para a pesquisa em ensino de ciências. Revista Pesquisa Qualitativa, v. 5, n. 9, 2017, p. 569-584.

SILVA, R. N.; BELO, M. L. M. Experiências e reflexões de monitoria: contribuição ao ensinoaprendizagem. Scientia Plena, v. 8, n. 7, 2012, p. 15-18.

SILVA, L. B; PAULINO, W. M; MACEDO, O. J. V. Contribuições da monitoria no processo de construção da identidade docente. In: CONGRESSO NACIONAL DE EDUCAÇÃO, 2, Campina Grande/PB. Atas... Campina Grande/PB: 2015. Disponível em:

<http://www.editorarealize.com.br/revistas/conedu/anais.php>. Acesso em: 23 mar. 2020.

SCHMITT, M. D.; RIBEIRO, M. C.; ADAMY, E. K.; BRUM, M. L. B.; ZANOTELLI, S. S. Contribuições da monitoria em semiologia e semiotécnica para a formação do enfermeiro: relato de experiência. Revista Cidadania em Ação: Extensão e Cultura, v. 7, n. 3, 2013, p. 18.

SOARES, M. A. A.; SANTOS, K. F. A monitoria como subsídio ao processo de ensinoaprendizagem: 0 caso da disciplina Administração Financeira no CCHSAUFPB. In: Encontro de iniciação à docência, 2008. Disponível em:

<http://www.prac.ufpb.br/anais/xenex_xienid/xi_enid/monitoriapet/ANAIS/Area4/4CCHSADCSAMT04.pdf>. Acesso: 25 fev. 2020.

SOUZA, R. O.; GOMES, A. R. A eficácia da monitoria no processo de aprendizagem visando a permanência do aluno na IES. Revista Interdisciplinar do Pensamento Científico, v. 1, n. 2, 2015, p. 35-40.

UNIDERP. Regulamentação do programa de monitoria de ensino da Universidade para o Desenvolvimento do Estado e da Região do Pantanal.

Disponível em: http://www.uniderp.br/ver_pagina.aspx? Acesso em: 12 mar. 2020.

VICENZI, C. B.; CONTO, F.; FLORES, M. E.; ROVANI, G.; FERRAZ, S. C. C.; MAROSTEGA, M. G. A monitoria e seu papel no desenvolvimento da formação acadêmica. Revista Ciência em Extensão, v. 12, n. 3, 2016, p. 88-94. 